\title{
NEUROSCIENCE
}

\section{A full fly connectome}

Zhang, Z. et al. Cell 174, 730-743 (2018).

Neuroscientists know little about how brain processes generate complex behaviors and functions such as learning and memory. Tracing neuronal circuitry will undoubtedly be a key part of solving this mystery, but mapping the connections between individual neurons requires an immense amount of high resolution images. A neuron's tangle of branches can contain tiny processes, 100 nanometers or less in diameter, that can't be comprehensively seen with a light microscope. "Right now the only way to make these maps for all neurons and synapses in a given piece of brain tissue is to use a technique called volume electron microscopy," says Davi Bock, a group leader at Janelia Research Campus.

In a recent paper in Cell, Bock and his team report using EM to create the first comprehensive map of all the neurons in the adult brain of Drosophila melanogaster. "This class of data has really been missing from the field of neurobiology," he says. "It doesn't answer all the questions by itself, but having a wiring diagram at that level of detail can't help but be useful."

Researchers have created comprehensive connectomes of a few small organisms, such as the nematode Caenorhabditis elegans and the fruit fly larva, but the 100,000 neuron brain of an adult fruit fly was thought to be out of reach. Bock's group began building a custom EM camera array as well as a stage that could quickly move the sample for highhigh-throughput image acquisition when he first arrived at Janelia in 2011. The group settled on the adult fly brain as the starting data-set and brought together a team of collaborators to optimize sample preparation and develop the needed software. "Stitching all these images into a coherent threedimensional volume was a huge problem at this scale," Bock says.

The 7,050 sections of fly brain that make up the map consist of roughly 21 million images-a 106 terabyte dataset. "We're one or two orders of magnitude larger than anything that's ever been done before at this resolution," Bock says. His group used the map to examine a fly brain region called the mushroom body, which stores associative olfactory memories. The unprecedented resolution revealed that cells in a subregion called the calyx made connections to a previously unobserved cell type.

The adult fly map is freely accessible online at temca2data.org or through a link at www.virtualflybrain.org. Already, some 20 labs are using the tool, says Bock. His lab and collaborators are now using deep learning techniques to do some early data processing and to speed up circuit mapping, so that users don't have to wade through raw data.

\section{Alla Katsnelson}

Published online: 23 October 2018

https://doi.org/10.1038/s41684-018-0183-8

\section{InSTECH}

\section{Oral gavage, refined.}

Our plastic feeding needles have flexible tubes with soft tips to reduce trauma, and they're disposable to eliminate the hassle of cleaning.

Available in a range of sizes for mice, rats and other rodents. www.instechlabs.com
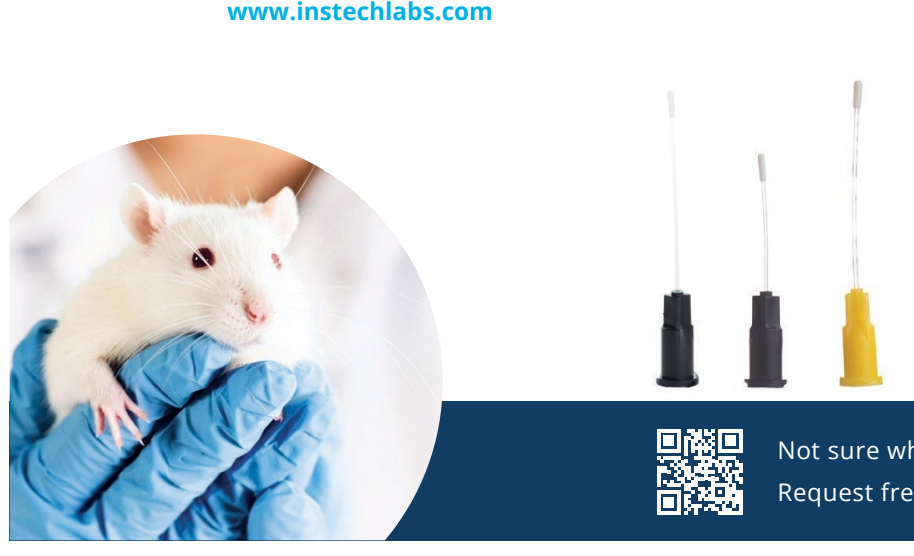